\title{
Electron Chemical Potential Measured for Graphene
}

\author{
Researchers demonstrate a method for measuring the chemical potential \\ in a many-electron system, providing a way to validate numerical \\ calculations.
}

By Marric Stephens

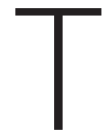

he answers to many questions in condensed-matter physics are hidden in the interactions that occur in quantum many-body systems. Such systems are notoriously difficult to simulate, with numerically tractable solutions existing under very limited circumstances.

Unfortunately for physicists, the calculable parameters of many-body systems tend to be experimentally inaccessible, making it difficult to test theories empirically. Now, Fangyuan Yang at the University of California, Santa Barbara, and colleagues have measured one previously inaccessible quantity-chemical potential-allowing precise benchmarking of numerical techniques [1].

In their experiments, the team probed a graphene-based 2D electron gas. The electron chemical potential of a system is the energy required to add or remove an electron from it. Together with the electron density, the chemical potential governs how the electrons in the system are arranged.

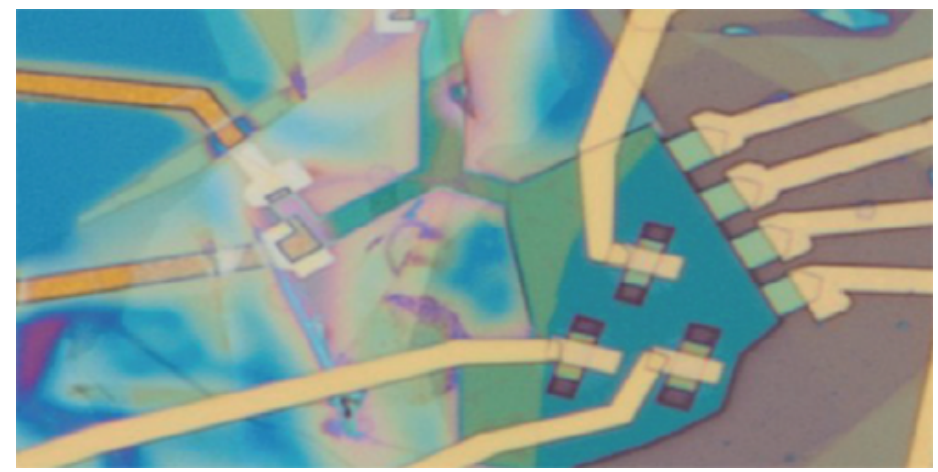

Credit: F. Yang et al. [1]
To perform their measurement, Yang and colleagues created a stacked structure of alternating graphene and boron nitride sheets, which they then subjected to a strong magnetic field. The field caused one electron from every few thousand of the carbon atoms in the graphene sheets to move in circular orbits called Landau levels.

Landau levels are quantized and each hosts a finite number of electrons; the fullness of a given level determines the system's electron density. Keeping the electron density in the top graphene layer constant, the researchers applied a voltage to the bottom layer, changing that layer's chemical potential and inducing an electric field. They then calculated this chemical potential by measuring how the induced electric field affected the conductivity of the other graphene layer. Such measurements have been attempted before, but only recently have nanofabrication techniques achieved the precision required to produce a successful experiment.

Marric Stephens is a Corresponding Editor for Physics based in Bristol, UK.

\section{REFERENCES}

1. F. Yang et al., "Experimental determination of the energy per particle in partially filled Landau levels," Phys. Rev. Lett. 126, 156802 (2021). 